\title{
Frailty and hospitalization-associated disability after pneumonia: A prospective cohort study
}

Chan Mi Park ${ }^{1 \dagger}$, Wonsock Kim ${ }^{1 \dagger}$, Hye Chang Rhim²${ }^{2}$, Eun Sik Lee ${ }^{1}$, Jong Hun Kim ${ }^{3 *}$ (D) Kyung Hwan Cho ${ }^{1}$ and Dae Hyun Kim ${ }^{4}$

\begin{abstract}
Background: Pneumonia is a major cause of morbidity and mortality in older adults. The role of frailty assessment in older adults with pneumonia is not well defined. Our purpose of the study was to investigate 30-day clinical course and functional outcomes of pneumonia in older adults with different levels of frailty.
\end{abstract}

Methods: A prospective cohort was conducted at a university hospital in Seoul, Korea with 176 patients who were 65 years or older and hospitalized with pneumonia. A 50-item deficit-accumulation frailty index (FI) (range: 0-1; robust $<0.15$, pre-frail $0.15-0.24$, mild-to-moderately frail $0.25-0.44$, and severely frail $\geq 0.45$ ) and the pneumonia severity CURB-65 score (range: 0-5) were measured. Primary outcome was death or functional decline, defined as worsening dependencies in 21 daily activities and physical tasks in 30 days. Secondary outcomes were intensive care unit admission, psychoactive drug use, nasogastric tube feeding, prolonged hospitalization (length of stay > 15 days), and discharge to a long-term care institution.

Results: The population had a median age 79 (interquartile range, 75-84) years, 68 (38.6\%) female, and 45 (25.5\%) robust, 36 (47.4\%) pre-frail, 37 (21.0\%) mild-to-moderately frail, and 58 (33.0\%) severely frail patients. After adjusting for age, sex, and CURB-65, the risk of primary outcome for increasing frailty categories was $46.7 \%, 61.1 \%, 83.8 \%$, and $86.2 \%$, respectively $(p=0.014)$. The risk was higher in patients with frailty $(\mathrm{FI} \geq 0.25)$ than without $(\mathrm{FI}<0.25)$ among those with CURB-65 $0-2$ points ( $75 \%$ vs. $52 \% ; p=0.022$ ) and among those with CURB-65 3-5 points (93\% vs. $65 \% ; p=0.007)$. In addition, patients with greater frailty were more likely to require nasogastric tube feeding (robust vs. severe frailty: $13.9 \%$ vs. $60.3 \%$ ) and prolonged hospitalization (18.2\% vs. $50.9 \%$ ) and discharge to a long-term care institution ( $4.4 \%$ vs. $59.3 \%$ ) ( $p<0.05$ for all). Rates of intensive care unit admission and psychoactive drug use were similar.

Conclusions: Older adults with frailty experience high rates of death or functional decline in 30 days of pneumonia hospitalization, regardless of the pneumonia severity. These results underscore the importance of frailty assessment in the acute care setting.

Keywords: Frailty, Pneumonia, Disability, Hospitalization-associated disability

\footnotetext{
* Correspondence: smonti1976@hotmail.com

Chan Mi Park and Wonsock Kim are equally contributed to the work.

${ }^{3}$ Division of Infectious Diseases, Department of Internal Medicine, CHA

Bundang Medical Center, CHA University, 59 Yatap-ro, Bundang-gu,

Seongnam 13496, Republic of Korea

Full list of author information is available at the end of the article
}

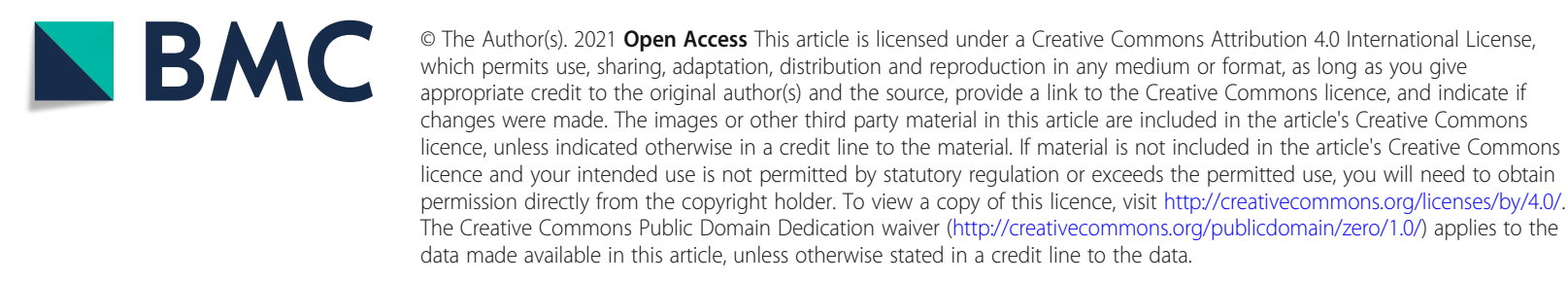




\section{Background}

Pneumonia is a major cause of morbidity and mortality in older adults [1]. In the United States, 5.6 million new cases are reported every year, and it accounts for high mortality and annual medical costs exceeding $\$ 10$ billion [2]. In Asia, pneumonia is responsible for almost 1 million adult deaths every year [3]. In Japan, pneumonia affected 1.8 million older Japanese and was the fifth leading cause of death in 2017 [4]. In Korea, it ranked fourth leading cause of death with a mortality rate of $11.6 \%[5,6]$ and the annual medical costs of $\$ 400$ million $[6,7]$.

Previous studies suggest that many patients with pneumonia have persistent symptoms and poor physical health after 30 days of diagnosis [8,9]. Older adults are particularly at high risk for poor outcomes [10], but the determinants of poor outcomes that are specific to this population have not been well characterized. Clinical risk stratification tools, such as CURB-65 [11] or Pneumonia Severity Index [9], predict mortality based on demographic information, comorbidities, or physiological parameters. However, these tools do not consider frailty - a clinical state of reduced physiologic reserve and increased vulnerability to poor health outcomes [12] - that is germane to clinical management of older adults. The prevalence of frailty ranges from $10 \%$ in the community [13] to more than $50 \%$ in the nursing homes [14] and hospitals [15], and it has been associated with poor health outcomes in the primary care [16], acute hospital [17], and critical care setting [18]. Therefore, assessing frailty on admission may provide information about patients' vulnerability and prognosis that is not captured by the pneumonia severity and is useful to deliver patient-centered care to improve recovery.

We conducted a prospective cohort study to evaluate the association of frailty with 30-day clinical and functional outcomes in older adults hospitalized with pneumonia. We hypothesized that frailty assessed on admission would be associated with mortality or functional decline at 30 days, independently of a pneumoniaspecific risk score. We also examined key care process measures during the acute hospitalization.

\section{Methods}

\section{Study design and population}

This prospective cohort study was approved by the Institutional Review Board and written informed consent was obtained from all patients or their proxy. Between October 2019 and June 2020, we approached consecutive patients who were 65 years or older and hospitalized with pneumonia at University Hospital, Seoul, Korea (see Flow Diagram in Fig. 1). Pneumonia was diagnosed based on symptoms (e.g., fever, cough, sputum, and dyspnea) plus an infiltrate on chest radiograph. During our study period, patients diagnosed with the novel Coronavirus Disease 2019 (COVID-19) were transferred to government-designated hospitals and only those with negative COVID-19 tests were admitted to our hospital. Among 265 patients screened, 89 were excluded for the following reasons: (1) patient declined $(n=56)$; (2) research team was unavailable $(n=14)$; $(3)$ informed consent could not be obtained from the patient or his/her proxy $(n=14)$; and (4) change in diagnosis after admission $(n=5)$. Finally, 176 patients were included in our study.

\section{Baseline assessment}

Study clinicians (CMP, WK, ESL) evaluated patients for medical comorbidities, self-reported functional status, cognitive function, nutritional status, and social support on admission. Self-reported functional status 30 days prior to admission was assessed by asking patients or their proxy about the ability to perform 21 daily activities and physical tasks without another person's assistance: 7 activities of daily living (feeding, dressing, grooming, ambulating, transferring, bathing, and toileting), 7 instrumental activities of daily living (doing housework, making telephone calls, using transportation or driving, shopping, cooking, taking medications, and managing money), and 7 activities in the Nagi and Rosow-Breslau scales (pulling or pushing a large object, lifting $5 \mathrm{~kg}$, walking up and down a flight of stairs, walking $1 \mathrm{~km}$, writing or handling small objects, reaching arms above shoulder, and stooping, crouching, or kneeling) $[19,20]$. Mobility impairment was defined as inability to ambulate in the house, walk $1 \mathrm{~km}$, or walk up and down a flight of stairs. Cognition was assessed using Mini-Mental State Examination (MMSE) or the AD8 questionnaire [21] if a patient was unable to participate in MMSE. Cognitive impairment was defined as having a dementia diagnosis, MMSE $<19$ points [22], or AD8 $\geq 3$ points [21]. Study clinicians (CMP, HCR) reviewed medical records to extract sociodemographic characteristics, admission source (nursing home vs. home), medical comorbidities, vital signs, body mass index, and laboratory test results (e.g., serum albumin).

\section{Measurements of frailty, pneumonia severity, and comorbidity burden}

A deficit-accumulation frailty index (FI) [23] was calculated using 50 items from baseline assessment: 26 comorbidities, polypharmacy ( $\geq 5$ prescription drugs), self-reported ability to perform 21 activities listed above, weight loss $>5 \mathrm{~kg}$ in past year, body mass index $<21 \mathrm{~kg} /$ $\mathrm{m}^{2}$, and serum albumin $<3.5 \mathrm{~g} / \mathrm{L}[24,25]$. MMSE was excluded from the FI calculation due to low completion rate $(38.6 \%)$. Based on FI (range: $0-1)$, patients were classified into robust $(<0.15)$, pre-frail $(0.15-0.24)$, mild- 


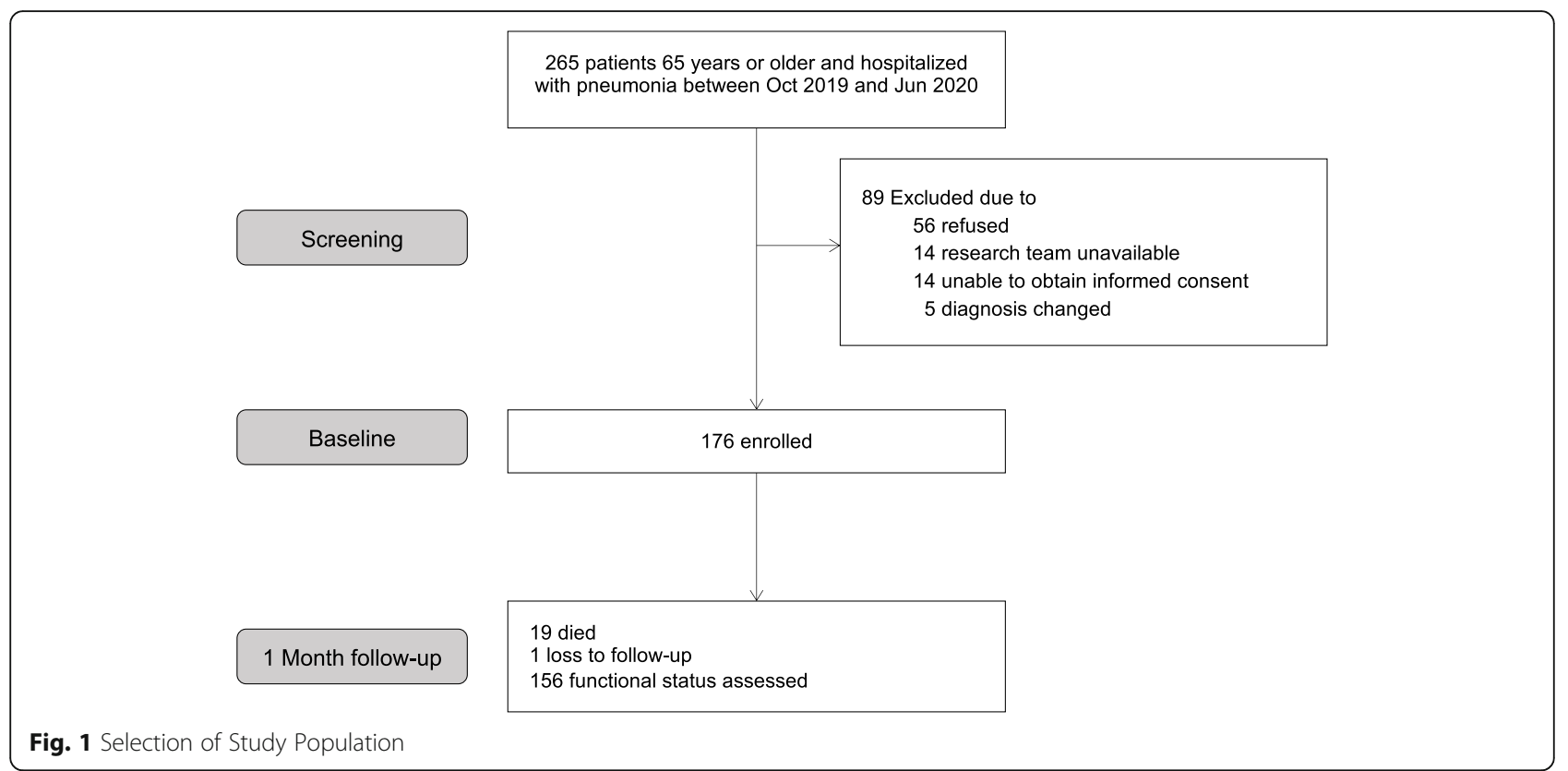

to-moderately frail $(0.25-0.44)$, and severely frail $(\geq 0.45)$ categories. Pneumonia severity was calculated using CURB-65 score (range: 0-5), which includes confusion, uremia, elevated respiratory rate, hypotension, and $\geq 65$ years of age [11]. The Gagne comorbidity index (range: $0-24$ ) was used to quantify the comorbidity burden [26].

\section{Outcome assessment}

At 1 month after baseline assessment, study clinicians (CMP, WK) conducted telephone interviews with patients or their proxy to assess self-reported functional status. Except 1 patient who was lost to follow-up and 19 patients who died, we were able to interview 156 patients. A disability score (range: $0-21$ ) was calculated as the total number of activities requiring another person's assistance. Primary outcome was a composite endpoint of death or functional decline, defined as any increase in the disability score between baseline and 1 month. As secondary patient outcomes, we examined death and functional decline at 30 days separately. As secondary process outcomes, we assessed intensive care unit (ICU) admission, psychoactive drug (antipsychotics, benzodiazepines, or hypnotics) use, nasogastric tube feeding, prolonged hospitalization (length of stay $>15$ days), and discharge to long-term care institution.

\section{Statistical analysis}

We compared baseline characteristics of patients across frailty categories using chi-square test for categorical variables and analysis of variance or Kruskal-Wallis test for continuous variables. The risks of primary and secondary outcomes were compared across frailty categories using chi-square test and logistic regression to estimate the odds ratio (OR) and $95 \%$ confidence interval (CI) adjusting for age, sex, and CURB-65 score (3-5 vs. $0-2$ points). Patients with the maximum disability at baseline were excluded from analysis for primary outcome and functional decline. Those who were admitted from a nursing home were excluded from analysis for long-term care institutionalization. We also examined the prevalence of each disability at baseline and 30 days later by frailty level on admission. To illustrate the importance of pneumonia severity and frailty, we estimated age- and sex-adjusted risk of primary outcome by CURB-65 (3-5 vs. 0-2 points) and frailty category (frail $[F I \geq 0.25]$ vs. non-frail $[F I<0.25]$ ) from a logistic model that included age, sex, CURB-65, frailty category, and the interaction term between CURB-65 and frailty categories. Lastly, in order to test the association between FI and CURB-65, spearman's correlation was calculated. Analysis was performed using Stata version 16 (StataCorp, LLC, College Station, Texas). A 2-sided $p$-value $<$ 0.05 was considered statistically significant.

\section{Results}

\section{Characteristics of study population}

The FI ranged from 0.02 to 0.65 , with 45 (25.5\%) robust, 36 (47.4\%) pre-frail, 37 (21.0\%) mild-to-moderately frail, and $58(33.0 \%)$ severely frail patients (Table 1). The study population had a median age of 79 (interquartile range [IQR], 75-84) years, 68 (38.6\%) female, and 35 $(19.9 \%)$ patients admitted from a nursing home. Generally, patients with greater frailty levels were older (median age [IQR] for robust vs. severe frailty: 77 [73-82] 
vs. 81 [75-84] years), admitted from a nursing home (0 [0\%] vs. 31 [53.5\%]), and have more severe pneumonia (CURB-65 score 3-5 points: 10 [22.2\%] vs. 36 [62.1\%]) and higher comorbidity burden (median Gagne index [IQR]: 2 [1,2,3] vs. $4[3,4,5,6])$. Increasing frailty was associated with stroke (robust vs. severe frailty: 8 [17.8\%] vs. $28[48.3 \%]$ ), disability (ADL: 0 [0\%] vs. 58 [100\%] and IADL: 3 [6.7\%] vs. 58 [100\%]), mobility impairment (7 [15.6\%] vs. 58 [100\%]), and cognitive impairment (7 [15.6\%] vs. 58 [100\%]) and lower body mass index (mean [standard deviation]: 22.4 [3.6] vs. 18.6 [3.8] kg/ $\mathrm{m}^{2}$ ). The spearman's correlation between FI and CURB65 was $0.34 ; p<0.001$, indicating a weak positive relationship.

\section{Frailty and patient outcomes at $\mathbf{3 0}$ days}

Among 147 patients who did not have the maximum disability on admission, 99 (67.4\%) developed the primary outcome (19 deaths and 84 with functional decline). The risk of primary outcome increased with the frailty level on admission, which remained statistically significant after adjusting for age, sex, and CURB-65 (Table 2): $46.7 \%$ for robust group, $61.1 \%$ for pre-frail group (adjusted OR [95\% CI], 1.46 [0.58-3.69]), $83.8 \%$ for mild-to-moderate frailty group (3.95 [1.31-11.89]) and $86.2 \%$ for severe frailty group (5.34 [1.54-18.49]). Similar patterns were observed for the individual outcomes of death and functional decline, but the association was not statistically significant for death due to low event rates.

The prevalence of each disability generally increased over 30 days across all frailty categories, except for severely frail patients who had high prevalence of preexisting disabilities (Fig. 2). At 30 days, a large proportion of patients were unable to walk $1 \mathrm{~km}$ (from robust to severely frail category: $40.5 \%, 77.1 \%, 96.8 \%$, and $100.0 \%)$ or walk up and down a flight of stairs $(26.2 \%$, $65.7 \%, 83.9 \%, 100.0 \%)$. Many patients needed help with ADLs, such as bathing or shower (from robust to severely frail category: $16.7 \%, 31.4 \%, 90.3 \%$, and $100.0 \%$ ) and toileting $(9.5 \%, 22.9 \%, 58.1 \%, 100.0 \%)$.

When we examined the risk of death or functional decline at 30 days by frailty level and pneumonia severity determined using CURB-65 on admission (Fig. 3), we found that frail patients had higher risk than non-frail patients among those with low CURB-65 scores (75\% vs. $52 \% ; p=0.022$ ) and among those with high CURB65 scores $(93 \%$ vs. $65 \% ; p=0.007)$. The interaction between frailty level and CURB-65 category was not statistically significant on the multiplicative scale $(p=$ 0.312 ), which means that the association of frailty with the primary outcome was constant across the pneumonia severity category.

\section{Frailty and process outcomes}

After adjusting for age, sex, and CURB-65, patients with greater frailty were more likely to require nasogastric tube feeding (robust vs. severe frailty: 5 [13.9\%] vs. 35 [60.3\%]) and prolonged hospitalization (8 [18.2\%] vs. 29 [50.9\%]) and be newly discharged to a long-term care institution (2 [4.4\%] vs. 16 [59.3\%]) (Table 2). However, new psychoactive drug use or ICU stay did not significantly differ by frailty level after adjustment.

\section{Discussion}

In our prospective cohort of 176 older patients hospitalized with pneumonia, frailty on admission was associated with death or functional decline at 30 days, independently of the pneumonia severity. In particular, 9 out of 10 patients with frailty and high CURB-65 score and 3 out of 4 patients with frailty and low CURB-65 score died or experienced functional decline at 30 days. The risk of nasogastric tube insertion, prolonged hospitalization, and new long-term care institutionalization was also higher for frail patients. These findings underscore the importance of assessing frailty, in addition to the pneumonia severity, to accurately inform patients and their family about prognosis and the ongoing care needs after a pneumonia hospitalization.

It is well accepted that older adults with frailty are more vulnerable than non-frail patients to the negative effect of acute stressors [12, 27], yet little is known about the relationship of frailty, severity of acute illness, and functional recovery after an acute illness [27]. A few studies have examined the association of frailty with clinical outcomes, such as mortality, ICU length of stay and readmission rates in the setting of acute illness [28, 29]. However, these studies did not assess functional status, which is a key determinant of older adults' independence and quality of life after hospitalization [30]. Moreover, the inter-relationship between frailty and the severity of acute illness on outcomes has not been fully explored. Although CURB-65 score predicts 30-day mortality and helps decisions about the level of care [11], recent studies reported its suboptimal performance for older adults [31, 32], particularly those 85 years or older [33]. Our study builds upon these previous studies by showing that both FI and pneumonia severity are important predictors of poor clinical and functional outcomes in older adults with pneumonia.

The current study adds to the literature on the feasibility of measuring frailty in the acute care setting. In previous studies of hospitalized patients, Clinical Frailty Scale (CFS), FI, and frailty phenotype were commonly used [27]. In the acute care setting, the simple CFS, and the 5-item FRAIL questionnaire were used more often than FI and frailty phenotype, which typically required more time and modifications to the original definition 
Table 1 Characteristics of Older Patients Hospitalized with Pneumonia

\begin{tabular}{|c|c|c|c|c|c|c|}
\hline \multirow[t]{2}{*}{ Characteristics } & \multirow[t]{2}{*}{ Total } & \multicolumn{4}{|c|}{ Frailty Category } & \multirow[t]{2}{*}{$P$ value } \\
\hline & & Robust & Pre-frailty & Mild-to-moderate frailty & Severe frailty & \\
\hline Sample Size & 176 & 45 & 36 & 37 & 58 & NA \\
\hline Frailty Index, median (IQR) & $0.31(0.14,0.50)$ & $0.10(0.06,0.12)$ & $0.18(0.16,0.20)$ & $0.35(0.31,0.41)$ & $0.54(0.50,0.58)$ & $<0.001$ \\
\hline Age, years, median (IQR) & $79(75,84)$ & $77(73,82)$ & $80(74,84)$ & $81(77,86)$ & $81(75,84)$ & 0.035 \\
\hline Female, n (\%) & 68 (38.6) & $14(31.1)$ & $14(38.9)$ & $17(46.0)$ & $23(39.7)$ & 0.586 \\
\hline Nursing Home Resident, n (\%) & 35 (19.9) & $0(0)$ & $1(2.8)$ & $3(8.1)$ & $31(53.5)$ & $<0.001$ \\
\hline CURB-65 Score 3-5 & $81(46.0)$ & $10(22.2)$ & 14(38.9) & $21(56.8)$ & $36(62.1)$ & $<0.001$ \\
\hline Gagne Index, median (IQR) & $3(1,4)$ & $2(1,3)$ & $2(1,4)$ & $3(1,5)$ & $4(3,6)$ & $<0.001$ \\
\hline Cardiovascular Disease, n (\%) & $48(27.3)$ & $11(24.4)$ & $9(25.0)$ & $14(37.8)$ & $14(24.1)$ & 0.450 \\
\hline Diabetes, n (\%) & $64(36.4)$ & $12(26.7)$ & $12(33.3)$ & $17(46.0)$ & $23(39.7)$ & 0.294 \\
\hline COPD, n (\%) & $28(15.9)$ & $6(13.3)$ & $8(22.2)$ & $9(24.3)$ & $5(8.6)$ & 0.135 \\
\hline Stroke, n (\%) & $49(27.8)$ & $8(17.8)$ & $3(8.3)$ & $10(27.0)$ & $28(48.3)$ & $<0.001$ \\
\hline ADL Dependency, n (\%) & $84(47.7)$ & $0(0.0)$ & $2(5.6)$ & $24(64.9)$ & $58(100.0)$ & $<0.001$ \\
\hline IADL Dependency, n (\%) & $107(60.8)$ & $3(6.7)$ & $10(27.8)$ & $36(97.3)$ & $58(100.0)$ & $<0.001$ \\
\hline Mobility Impairment, n (\%) & $124(70.5)$ & $7(15.6)$ & $24(66.7)$ & 35 (94.6) & $58(100.0)$ & $<0.001$ \\
\hline Cognitive Impairment, n (\%) & $132(75.0)$ & $37(82.2)$ & $21(58.3)$ & $26(70.3)$ & $48(82.8)$ & 0.031 \\
\hline $\mathrm{BMI}, \mathrm{kg} / \mathrm{m}^{2}$, median (IQR) & $22.0(4.6)$ & $22.4(3.6)$ & $24.7(5.1)$ & $22.0(3.9)$ & $18.6(3.8)$ & $<0.001$ \\
\hline Weight Loss, n (\%) & $42(23.9)$ & $8(17.8)$ & $5(13.9)$ & $10(27.0)$ & $19(32.8)$ & 0.132 \\
\hline
\end{tabular}

Abbreviations: $A D L$ activities of daily living, $B M I$ body mass index, COPD chronic obstructive pulmonary disease, IADL instrumental activities of daily living, IQR interquartile range

Table 2 Frailty and Outcomes in Older Patients Hospitalized with Pneumonia

\begin{tabular}{|c|c|c|c|c|c|c|}
\hline \multirow[t]{2}{*}{ Outcomes } & \multicolumn{5}{|c|}{ Number of Outcome Events (\%) and OR $(95 \% \mathrm{Cl})^{\mathrm{a}}$} & \multirow[t]{2}{*}{$P$ value } \\
\hline & Total & Robust & Pre-frailty & Mild-to-moderate frailty & Severe frailty & \\
\hline \multicolumn{7}{|l|}{ Primary Outcome } \\
\hline \multirow[t]{2}{*}{ Death or functional decline at 30 days $s^{b}$} & $99(67.4)$ & $21(46.7)$ & $22(61.1)$ & $31(83.8)$ & $25(86.2)$ & $<0.001$ \\
\hline & NA & Reference & $1.46(0.58-3.69)$ & $3.95(1.31-11.89)$ & $5.34(1.54-18.49)$ & 0.014 \\
\hline \multicolumn{7}{|l|}{ Secondary patient outcomes } \\
\hline \multirow[t]{2}{*}{ Death at 30 days } & $19(10.8)$ & $3(6.7)$ & $1(2.8)$ & $6(16.2)$ & $9(15.5)$ & 0.129 \\
\hline & NA & Reference & $0.38(0.37-3.84)$ & $2.36(0.51-10.94)$ & $2.27(0.54-9.62)$ & 0.281 \\
\hline \multirow[t]{2}{*}{ Functional decline at 30 days $^{\mathrm{b}}$} & $84(63.6)$ & $18(42.9)$ & $21(60.0)$ & $25(80.7)$ & $20(83.3)$ & 0.001 \\
\hline & NA & Reference & $1.63(0.63-4.20)$ & $3.84(1.24-11.86)$ & $5.33(1.50-19.02)$ & 0.022 \\
\hline \multicolumn{7}{|l|}{ Secondary process outcomes } \\
\hline \multirow[t]{2}{*}{ Intensive care unit stay } & $38(21.8)$ & $4(8.9)$ & $8(22.2)$ & $9(24.3)$ & $17(30.4)$ & 0.074 \\
\hline & NA & Reference & $2.51(0.67-9.44)$ & $2.44(0.64-9.27)$ & $3.15(0.92-10.73)$ & 0.336 \\
\hline \multirow[t]{2}{*}{ Psychoactive drug use $^{c}$} & $41(26.5)$ & $10(22.2)$ & $7(22.6)$ & $13(41.9)$ & $11(22.9)$ & 0.189 \\
\hline & NA & Reference & $0.88(0.28-2.78)$ & $1.92(0.64-5.78)$ & $0.66(0.22-1.96)$ & 0.236 \\
\hline \multirow[t]{2}{*}{ Nasogastric tube feeding } & $50(28.4)$ & $3(6.7)$ & $5(13.9)$ & $7(18.9)$ & $35(60.3)$ & $<0.001$ \\
\hline & NA & Reference & $1.97(0.42-9.22)$ & $2.50(0.56-11.22)$ & $17.08(4.49-64.99)$ & $<0.001$ \\
\hline \multirow[t]{2}{*}{ Prolonged hospitalization ( $\geq 15$ days) } & $62(35.6)$ & $8(18.2)$ & $14(38.9)$ & $11(29.7)$ & $29(50.9)$ & 0.006 \\
\hline & NA & Reference & $2.72(0.95-7.82)$ & $1.58(0.52-4.80)$ & $3.72(1.38-9.98)$ & 0.039 \\
\hline \multirow[t]{2}{*}{ Discharge to a long-term care institution ${ }^{d}$} & $39(27.7)$ & $2(4.4)$ & $7(20.0)$ & $14(41.2)$ & $16(59.3)$ & $<0.001$ \\
\hline & NA & Reference & $4.56(0.83-24.87)$ & $9.79(1.88-51.00)$ & $25.11(4.70-134.07)$ & $<0.001$ \\
\hline
\end{tabular}

Abbreviations: $\mathrm{Cl}$ confidence interval, $N A$ not applicable, $O R$ odds ratio

${ }^{a}$ Adjusted for age, sex, and CURB-65 score

${ }^{b}$ Assessed for 147 patients who did not have the maximum disability on admission

${ }^{c}$ Assessed for 155 patients who were not receiving psychoactive drugs on admission

${ }^{\mathrm{d}}$ Assessed for 141 patients who were not admitted from a nursing home 

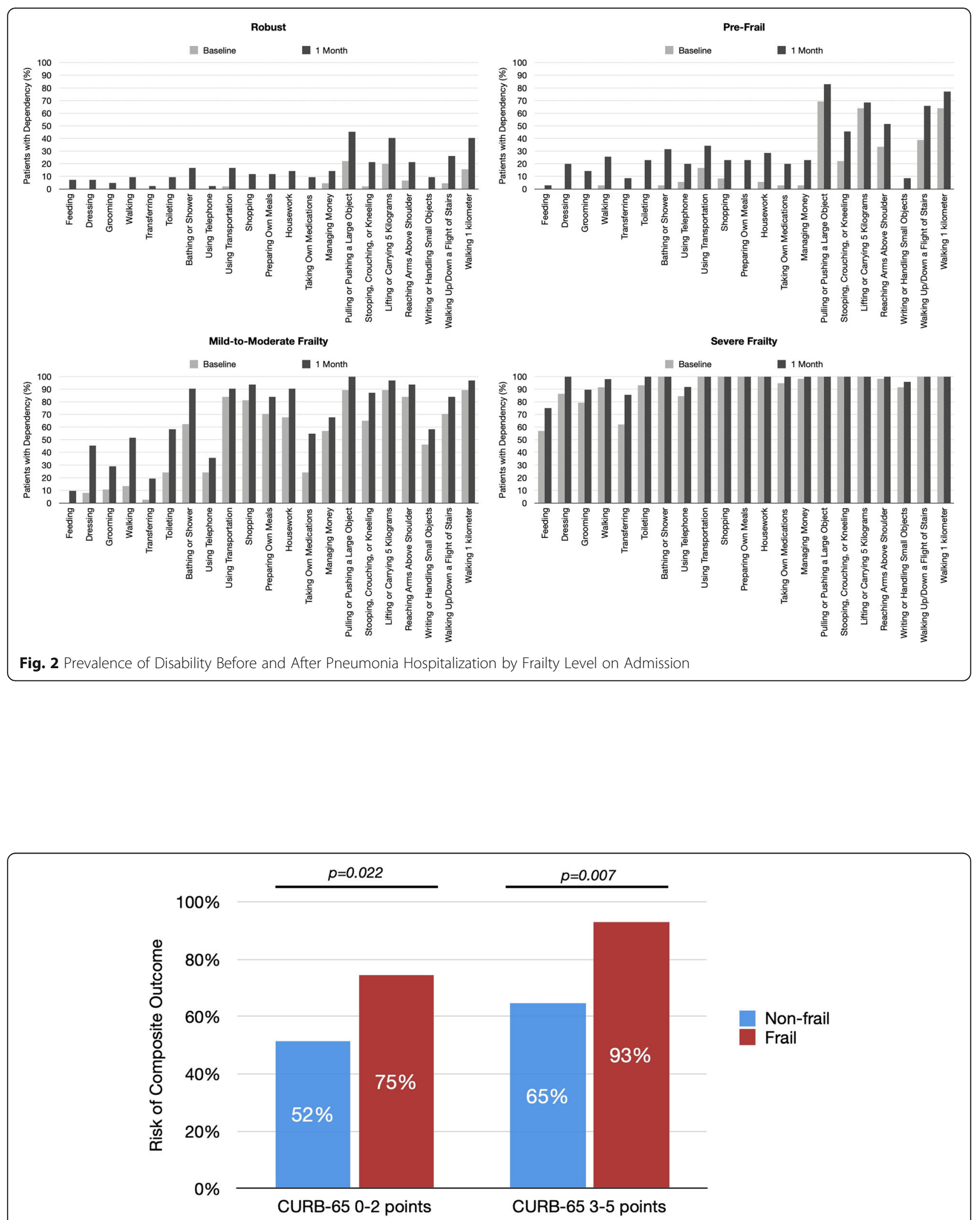

Fig. 3 Risk of Death or Functional Decline at 30 days by Frailty and Pneumonia Severity. The risk (vertical bar) of death or functional decline was presented for frail (red bar; frailty index $\geq 0.25$ ) vs non-frail (blue bar; frailty index $<0.25$ ) patients with low pneumonia severity (CURB-65 $0-2$ points) vs high pneumonia severity (CURB-65 3-5 points) 
[34-36]. Although simpler measures of frailty may be useful for rapid detection of who might be frail, a more sophisticated tool, such as comprehensive geriatric assessment (CGA)-based FI, should be followed to assess and confirm the severity of frailty for clinical management [37]. In our study, FI assessment and calculation took approximately $30-45 \mathrm{~min}$ at the patient's bedside with few missing items. While this may be considered time-consuming, use of routinely available electronic medical record or administrative claims data [38, 39], online calculators [25,40], or mobile applications [41] may shorten administration time and improve interpretation.

Moreover, our results underscore high incidence of hospitalization-associated disability in older adults after a pneumonia hospitalization. Such hospitalizationassociated disability occurs frequently among older adults after acute illness and sometimes they are irreversible [42-44]. Our study and several others [17, 45, 46] have shown that CGA can identify older adults at high risk for hospitalization-associated disability. Hospitalized older adults who received clinical care based on CGA were more likely to live at home and less likely to be admitted to a nursing home $[45,47]$. The assessment is an essential first step to identify frail patients but it also works as an intervention by delivering individualized treatments to patients $[17,47]$. According to our findings, preventative interventions for older patients with frailty should begin during the hospitalization period to avoid functional decline and promote independence. These interventions may include regular ambulation, encouraging performance of activities of daily living, and education for patients or their caregivers to assess noticeable change in functional status.

Major strengths of our study include prospective evaluation of a deficit-accumulation FI in the acute care setting, measurement of pneumonia severity, and high rates of 30-day follow-up assessment for functional status. Our study has a few limitations that deserve mention. First, the rates of death or functional decline in our cohort of older Koreans may not be generalizable to other populations in different health systems. Second, functional status was self-reported, rather than direct observation. However, the validity of self-reported functional status has been demonstrated [48], and we believe that telephone interview may be the most practical method to attain high response rates, especially during COVID-19 pandemic. Third, we only assessed outcomes over 30 days. Longer follow-up assessments are underway. Lastly, our frailty assessment did not include physical performance test and MMSE due to low completion rates in the acute care setting. Despite this missingness, we found that our FI was associated with the primary outcome. According to a recent study, missing clinical domains may affect the prevalence estimates of frailty but have little impact on the predictive ability [49].

\section{Conclusions}

In conclusion, our study shows high rates of death or functional decline at 30 days in older patients with frailty hospitalized with pneumonia, regardless of the pneumonia severity. Frailty assessment in the acute care setting is feasible and useful to accurately inform prognosis and care needs following hospitalization. Further research is warranted to test acute care and post-acute care interventions to prevent functional decline after pneumonia.

\section{Abbreviations \\ Fl: Frailty Index; OR: Odds ratio; Cl: Confidence interval; CFS: Clinical frailty scale; CGA: Comprehensive geriatric assessment}

\section{Acknowledgements}

This work was presented in part at the Gerontological Society of America (GSA) 2020 Annual Scientific Meeting.

\section{Authors' contributions}

Study design and concept: CMP, JHK, DHK. All authors have read and approved the manuscript. Acquisition, analysis, and interpretation of data: CMP, WK, HCR, ESL, JHK, KHC, DHK. Drafting of the manuscript: CMP. Critical revision of the manuscript for important intellectual content: CMP, WK, HCR, ESL, JHK, KHC, DHK. Statistical analysis: CMP, DHK. Administrative, technical, or material support: CMP, JHK, KHC. Study supervision: CMP, JHK, DHK.

\section{Funding}

None

\section{Availability of data and materials}

The datasets used and/or analyzed during the current study are available from Dr. Chan Mi Park and Dr. Jong Hun Kim on reasonable request.

\section{Ethics approval and consent to participate}

This prospective cohort study was approved by the Institutional Review Board of Korea University Medical Center, Seoul, Korea, and written informed consent was obtained from all patients prior to each assessment. (IRB No.2019AN0408) If a patient was deemed to lack capacity, we obtained informed consent from their legally authorized representative.

\section{Consent for publication}

Not applicable.

\section{Competing interests}

DHK provides paid consultative services to Alosa Health, a nonprofit educational organization with no relationship to any drug or device manufacturers. He also receives research grants from National Institute on Aging for unrelated projects. The other authors declare no competing interests.

\section{Author details}

${ }^{1}$ Department of Family Medicine, Korea University Anam Hospital, Korea University College of Medicine, Seoul, Republic of Korea. ${ }^{2}$ Harvard T.H Chan School of Public Health, Boston, MA, USA. ${ }^{3}$ Division of Infectious Diseases, Department of Internal Medicine, CHA Bundang Medical Center, CHA University, 59 Yatap-ro, Bundang-gu, Seongnam 13496, Republic of Korea. ${ }^{4} \mathrm{Hinda}$ and Arthur Marcus Institute for Aging Research, Hebrew SeniorLife, Harvard Medical School, Boston, MA, USA.

Received: 17 September 2020 Accepted: 24 January 2021

Published online: 05 February 2021

\section{References}

1. Callahan CM, Wolinsky FD. Hospitalization for pneumonia among older adults. J Gerontol A Biol Sci Med Sci. 1996;51(6):M276-82. 
2. Jain $S$, Self WH, Wunderink RG, Fakhran S, Balk R, Bramley AM, et al. Community-Acquired Pneumonia Requiring Hospitalization among U.S. Adults. N Engl J Med. 2015;373(5):415-27.

3. World Health Organization. Global burden of disease (GBD). Geneva: World Health Organization; 2008. http://www.who.int/healthinfo/global_burden_ disease/gbd/en/.

4. Song JH, Huh K, Chung DR. Community-Acquired Pneumonia in the AsiaPacific Region. Semin Respir Crit Care Med. 2016;37(6):839-54

5. Korean Statistics. Korean Statistical Information Service. Cause of Death Statistics; 2016. Available at: http://kostat.go.kr/portal/korea/kor_nw/2/6/1/ index.board?bmode $=$ read\&aSeq $=363268$.

6. Choi JY, Kim SW, Yoon SJ, Kang MG, Kim KI, Kim CH. Impact of frailty on donot-resuscitate orders and healthcare transitions among elderly Koreans with pneumonia. Clin Interv Aging. 2018;13:2237-45.

7. Yoo KH, Yoo CG, Kim SK, Jung JY, Lee MG, Uh ST, et al. Economic burden and epidemiology of pneumonia in Korean adults aged over 50 years. J Korean Med Sci. 2013;28(6):888-95.

8. Fine MJ, Stone RA, Singer DE, Coley CM, Marrie TJ, Lave JR, et al. Processes and outcomes of care for patients with community-acquired pneumonia: results from the Pneumonia Patient Outcomes Research Team (PORT) cohort study. Arch Intern Med. 1999;159(9):970-80.

9. Metlay JP, Fine MJ, Schulz R, Marrie TJ, Coley CM, Kapoor WN, et al. Measuring symptomatic and functional recovery in patients with community-acquired pneumonia. J Gen Intern Med. 1997;12(7):423-30.

10. Bloom ASSS, Steinbrink J, McClain MT. Utility of predictive tools for risk stratification of elderly individuals with all-cause acute respiratory infection. Infection. 2019;47(4):617-27.

11. Lim WS, van der Eerden MM, Laing R, Boersma WG, Karalus N, Town Gl, et al. Defining community acquired pneumonia severity on presentation to hospital: an international derivation and validation study. Thorax. 2003;58(5):377-82.

12. Clegg A, Young J, lliffe S, Rikkert MO, Rockwood K. Frailty in elderly people. Lancet. 2013;381(9868):752-62.

13. Collard RM, Boter H, Schoevers RA, Oude Voshaar RC. Prevalence of frailty in community-dwelling older persons: a systematic review. J Am Geriatr Soc. 2012;60(8):1487-92.

14. Kojima G. Prevalence of Frailty in Nursing Homes: A Systematic Review and Meta-Analysis. J Am Med Dir Assoc. 2015;16(11):940-5.

15. Richards SJG, D'Souza J, Pascoe R, Falloon M, Frizelle FA. Prevalence of frailty in a tertiary hospital: A point prevalence observational study. Plos one. 2019; 14(7):e0219083.

16. Carey EC, Covinsky KE, Lui LY, Eng C, Sands LP, Walter LC. Prediction of mortality in community-living frail elderly people with long-term care needs. J Am Geriatr Soc. 2008;56(1):68-75.

17. Conroy SP, Bardsley M, Smith P, Neuburger J, Keeble E, Arora S, et al. Health Services and Delivery Research. Comprehensive geriatric assessment for frail older people in acute hospitals: the HoW-CGA mixed-methods study. Southampton: NIHR Journals Library; 2019.

18. Le Maguet P, Roquilly A, Lasocki S, Asehnoune K, Carise E, Saint Martin M, et al. Prevalence and impact of frailty on mortality in elderly ICU patients: a prospective, multicenter, observational study. Intensive Care Med. 2014; 40(5):674-82.

19. Nagi SZ. An epidemiology of disability among adults in the United States. Milbank Mem Fund Q Health Soc. 1976;54(4):439-67.

20. Rosow I, Breslau N. A Guttman health scale for the aged. J Gerontol. 1966; 21(4):556-9.

21. Galvin JE, Roe CM, Xiong C, Morris JC. Validity and reliability of the AD8 informant interview in dementia. Neurology. 2006;67(11):1942-8.

22. Folstein MF, Folstein SE, McHugh PR. "Mini-mental state". A practical method for grading the cognitive state of patients for the clinician. J Psychiatr Res. 1975;12(3):189-98.

23. Rockwood K, Mitnitski A. Frailty defined by deficit accumulation and geriatric medicine defined by frailty. Clin Geriatr Med. 2011;27(1):17-26.

24. Kim DH, Afilalo J, Shi SM, Popma JJ, Khabbaz KR, Laham RJ, et al. Evaluation of Changes in Functional Status in the Year After Aortic Valve Replacement. JAMA Intern Med. 2019;179(3):383-91.

25. Senior Health Calculator. For Providers: Online Tool to Calculate Frailty Index (FI). Available at: https://www.bidmc.org/research/research-by-department/ medicine/gerontology/calculator.

26. Gagne JJ, Glynn RJ, Avorn J, Levin R, Schneeweiss S. A combined comorbidity score predicted mortality in elderly patients better than existing scores. J Clin Epidemiol. 2011;64(7):749-59.
27. Theou O, Squires E, Mallery K, Lee JS, Fay S, Goldstein J, et al. What do we know about frailty in the acute care setting? A scoping review. BMC Geriatr. 2018;18(1):139

28. Muscedere J, Waters B, Varambally A, Bagshaw SM, Boyd JG, Maslove D, et al. The impact of frailty on intensive care unit outcomes: a systematic review and meta-analysis. Intensive Care Med. 2017:43(8):1105-22.

29. Kundi H, Wadhera RK, Strom JB, Valsdottir LR, Shen C, Kazi DS, et al. Association of Frailty With 30-Day Outcomes for Acute Myocardial Infarction, Heart Failure, and Pneumonia Among Elderly Adults. JAMA Cardiol. 2019;4(11):1084-91.

30. Abdelbasset WK, Alsubaie SF, Tantawy SA, Elyazed TIA, Elshehawy AA. A cross-sectional study on the correlation between physical activity levels and health-related quality of life in community-dwelling middle-aged and older adults. Medicine. 2019;98(11):e14895.

31. Ma HM, Ip M, Woo J. Effect of age and residential status on the predictive performance of CURB-65 score. Intern Med J. 2015;45(3):300-4.

32. Ilg A, Moskowitz A, Konanki V, Patel PV, Chase M, Grossestreuer AV, et al. Performance of the CURB-65 Score in Predicting Critical Care Interventions in Patients Admitted With Community-Acquired Pneumonia. Ann Emerg Med. 2019;74(1):60-8.

33. Zhang ZX, Yong Y, Tan WC, Shen L, Ng HS, Fong KY. Prognostic factors for mortality due to pneumonia among adults from different age groups in Singapore and mortality predictions based on PSI and CURB-65. Singapore Med J. 2018;59(4):190-8.

34. Pugh RJ, Ellison A, Pye K, Subbe CP, Thorpe CM, Lone NI, et al. Feasibility and reliability of frailty assessment in the critically ill: a systematic review. Crit Care. 2018;22(1):49.

35. Rodriguez-Queralto O, Formiga F, Lopez-Palop R, Marin F, Vidan MT, Martinez-Selles M, et al. FRAIL Scale also Predicts Long-Term Outcomes in Older Patients With Acute Coronary Syndromes. J Am Med Dir Assoc. 2020; 21(5):683-7 e1.

36. Chua XY, Toh S, Wei K, Teo N, Tang T, Wee SL. Evaluation of clinical frailty screening in geriatric acute care. J Eval Clin Pract. 2020;26(1):35-41.

37. Dent E, Kowal P, Hoogendijk EO. Frailty measurement in research and clinical practice: A review. Eur J Intern Med. 2016:31:3-10.

38. Clegg A, Bates C, Young J, Ryan R, Nichols L, Ann Teale E, et al. Development and validation of an electronic frailty index using routine primary care electronic health record data. Age Ageing. 2016;45(3):353-60.

39. Kim DH. Measuring Frailty in Health Care Databases for Clinical Care and Research. Ann Geriatr Med Res. 2020;24(2):62-74.

40. CGA Toolkit Plus. Available at: https://www.cgakit.com/frailty-index.

41. Frailty Tool. Rapidly Evaluate Frailty and Individualize Care for Your Patient. Available at: http://frailtytool.com/.

42. Covinsky KE, Pierluissi E, Johnston CB. Hospitalization-associated disability: "She was probably able to ambulate, but I'm not sure". JAMA. 2011;306(16): 1782-93.

43. Loyd C, Markland AD, Zhang Y, Fowler M, Harper S, Wright NC, et al. Prevalence of Hospital-Associated Disability in Older Adults: A Meta-analysis. J Am Med Dir Assoc. 2020;21(4):455-61. e5.

44. Dharmarajan K, Han L, Gahbauer EA, Leo-Summers LS, Gill TM. Disability and Recovery After Hospitalization for Medical IIIness Among Community-Living Older Persons: A Prospective Cohort Study. J Am Geriatr Soc. 2020;68(3):486-95.

45. Ellis G, Gardner M, Tsiachristas A, Langhorne P, Burke O, Harwood RH, et al. Comprehensive geriatric assessment for older adults admitted to hospital. Cochrane Database Syst Rev. 2017;9:CD006211.

46. Hosler QP, Maltagliati AJ, Shi SM, Afilalo J, Popma JJ, Khabbaz KR, et al. A Practical Two-Stage Frailty Assessment for Older Adults Undergoing Aortic Valve Replacement. J Am Geriatr Soc. 2019;67(10):2031-7.

47. Parker SG, McCue P, Phelps K, McCleod A, Arora S, Nockels K, et al. What is Comprehensive Geriatric Assessment (CGA)? An umbrella review. Age Ageing. 2018;47(1):149-55

48. Young Y, Boyd CM, Guralnik JM, Fried LP. Does self-reported function correspond to objective measures of functional impairment? J Am Med Dir Assoc. 2010;11(9):645-53.

49. Shi SM, McCarthy EP, Mitchell S, Kim DH. Changes in Predictive Performance of a Frailty Index with Availability of Clinical Domains. J Am Geriatr Soc. 2020;68(8):1771-7

\section{Publisher's Note}

Springer Nature remains neutral with regard to jurisdictional claims in published maps and institutional affiliations. 